archives-ouvertes

\title{
Non-linear MHD modeling of edge localized mode cycles and mitigation by resonant magnetic perturbations
}

\author{
Marina Becoulet, Francois Orain, M Bécoulet, M Morales, G Huijsmans, G.
}

Dif-Pradalier, M Hoelzl, G Garbet, S. Pamela, E. Nardon, et al.

\section{To cite this version:}

Marina Becoulet, Francois Orain, M Bécoulet, M Morales, G Huijsmans, et al.. Non-linear MHD modeling of edge localized mode cycles and mitigation by resonant magnetic perturbations. Plasma Physics and Controlled Fusion, IOP Publishing, 2015, 57 (1), pp.014020. 10.1088/0741-3335/57/1/014020 . hal-02184569

\section{HAL Id: hal-02184569 \\ https://hal.archives-ouvertes.fr/hal-02184569}

Submitted on 16 Jul 2019

HAL is a multi-disciplinary open access archive for the deposit and dissemination of scientific research documents, whether they are published or not. The documents may come from teaching and research institutions in France or abroad, or from public or private research centers.
L'archive ouverte pluridisciplinaire HAL, est destinée au dépôt et à la diffusion de documents scientifiques de niveau recherche, publiés ou non, émanant des établissements d'enseignement et de recherche français ou étrangers, des laboratoires publics ou privés. 
Non-linear MHD modeling of edge localized mode cycles and mitigation by resonant magnetic perturbations

This content has been downloaded from IOPscience. Please scroll down to see the full text.

2015 Plasma Phys. Control. Fusion 57014020

(http://iopscience.iop.org/0741-3335/57/1/014020)

View the table of contents for this issue, or go to the journal homepage for more

Download details:

IP Address: 132.169.10.169

This content was downloaded on 28/01/2015 at 12:32

Please note that terms and conditions apply. 


\title{
Non-linear MHD modeling of edge localized mode cycles and mitigation by resonant magnetic perturbations
}

\author{
François Orain ${ }^{1}$, M Bécoulet ${ }^{1}$, J Morales ${ }^{1}, \mathrm{G}$ T A Huijsmans ${ }^{2}$, \\ G Dif-Pradalier ${ }^{1}$, M Hoelzl $^{3}$, X Garbet ${ }^{1}$, S Pamela ${ }^{4}$, E Nardon ${ }^{1}$, C Passeron ${ }^{1}$, \\ G Latu ${ }^{1}$, A Fil ${ }^{1}$ and $P$ Cahyna ${ }^{5}$ \\ ${ }^{1}$ CEA-IRFM, Cadarache, 13108 Saint-Paul-Lez-Durance, France \\ ${ }^{2}$ ITER Organization, Route de Vinon-sur-Verdon, CS 90 046, 13067 Saint-Paul-Lez-Durance, France \\ 3 Max Planck Institute for Plasma Physics, Boltzmannstr. 2, 85748 Garching, Germany \\ ${ }^{4}$ CCFE, Culham Science Centre, Oxon, Abingdon, Oxfordshire OX14 3DB, UK \\ 5 Institute of Plasma Physics ASCR, Za Slovankou 1782/3, 18200 Prague 8, Czech Republic \\ E-mail: francois.orain@cea.fr
}

Received 27 June 2014, revised 7 September 2014

Accepted for publication 22 September 2014

Published 28 November 2014

\begin{abstract}
The dynamics of a multi-edge localized mode (ELM) cycle as well as the ELM mitigation by resonant magnetic perturbations (RMPs) are modeled in realistic tokamak X-point geometry with the non-linear reduced MHD code JOREK. The diamagnetic rotation is found to be a key parameter enabling us to reproduce the cyclical dynamics of the plasma relaxations and to model the near-symmetric ELM power deposition on the inner and outer divertor target plates consistently with experimental measurements. Moreover, the non-linear coupling of the RMPs with unstable modes are found to modify the edge magnetic topology and induce a continuous MHD activity in place of a large ELM crash, resulting in the mitigation of the ELMs. At larger diamagnetic rotation, a bifurcation from unmitigated ELMs—at low RMP current- towards fully suppressed ELMs—at large RMP current-is obtained.
\end{abstract}

Keywords: ELM, RMP, MHD, cycles, mitigation, tokamaks, plasmas

(Some figures may appear in colour only in the online journal)

\section{Introduction}

Edge localized modes (ELMs) are magnetohydrodynamic (MHD) events occurring in high-confinement regime ( $\mathrm{H}$-mode) of tokamak plasmas. They are initiated with the growth of instabilities triggered by the large edge pressure gradient (socalled ballooning modes) or by the large edge current (so-called peeling modes) [1]. These instabilities generate a quasi-periodic relaxation of the edge plasma (also called pedestal), and represent a cyclical dynamics of the plasma. Experimentally, ELM precursors (possibly related to peeling-ballooning instabilities) are observed to grow and rotate in the pedestal in the electron diamagnetic direction, followed by the expulsion of filaments through the edge transport barrier [2, 3]. A possible understanding is that the ELM precursors are triggered when the peeling-ballooning stability boundary is crossed, and grow until the plasma non-linearly reorganizes; as a result, the pressure profile relaxes and filaments are expelled from the plasma. The relaxation brings back the plasma to a stable state, yet as the pedestal is rebuilt, the profiles steepen and the plasma is re-destabilized, inducing another ELM crash [4]. The heat flux reaching the divertor due to an ELM is foreseen to be likely to erode the divertor in ITER if the ELMs are not mitigated, thus the ELMs have to be controlled in ITER [5]. A promising control method is the application of resonant magnetic perturbations (RMPs) by dedicated coils. This method proved to be successful in either suppressing the ELMs in the DIII-D [6, 7] and KSTAR tokamaks [8] or in mitigating the ELM power in ASDEX Upgrade [9], JET [10], MAST [11] and NSTX [12], which validated its use for ITER operation. 
So far, only a single ELM crash could be reproduced in modeling in realistic geometry with non-linear MHD codes such as M3D [13], BOUT + + [14, 15], NIMROD [16] and JOREK [17-19]; however multi-ELM cycling regimes were not reproduced in modeling up to now. As for the ELM mitigation by RMPs, its observation in modeling is a new result. The reduced MHD code JOREK was recently extended to introduce the bifluid diamagnetic effects, the neoclassical friction and a source of toroidal rotation, in order to self-consistently describe the plasma flows. Also, RMPs were added in the code, the vacuum RMP field being set as boundary conditions for the perturbation of the magnetic flux. The full model is described in [20]. These improvements now enable to simulate both multi-ELM cycles-we show in this paper that the diamagnetic stabilization is a key parameter that allows for obtaining them-and the ELM mitigation or suppression by RMPs. Section 2 presents the modeling of ELM cycles in JET-like configuration. We show that a competition between the diamagnetic stabilization and the plasma destabilization by the growth of the pressure gradient due to the heat source generates the cyclical dynamics of the plasma relaxation and pedestal reconstruction. The near-symmetric repartition of the ELM power on the inner and outer divertor target plates due to the convection of the filaments by the diamagnetic drifts is also presented. In section 3, the mechanism of the ELM mitigation or suppression by RMPs is described. Under a certain RMP current threshold, the ELM mitigation is due to the continuous MHD activity produced by the non-linear interaction between RMPs and the unstable plasma modes. When the RMP current is sufficiently increased, the plasma is stabilized under the peeling-ballooning instability threshold and the ELMs are fully suppressed.

\section{ELM cycle studies}

ELM simulations are performed for JET-like plasma parameters and geometry, at low triangularity shape, similar to [20]: major radius $R_{0}=3 \mathrm{~m}$, minor radius $a=1 \mathrm{~m}$, toroidal magnetic field $B_{t}=2.9 \mathrm{~T}$ and safety factor $q_{95} \sim 3$. Established $\mathrm{H}$-mode experimental profiles are taken initially, with central electron density $n_{e, 0}=6 \times 10^{19} \mathrm{~m}^{-3}$ and central temperature $T_{e, 0}=5 \mathrm{keV}$. The pedestal density and temperature are $n_{e, \text { ped }}=3.8 \times 10^{19} \mathrm{~m}^{-3}$ and $T_{e, \text { ped }}=2.5 \mathrm{keV}$. The edge transport barrier is maintained by reducing the perpendicular diffusive coefficients of heat and particles in the pedestal. Ad hoc heat and particle sources are chosen to mimic e.g. the heating power due to neutral beam injection: the heat source follows the pedestal profile and the density source is constant over the plasma. These sources are chosen to ensure that the pedestal can reconstruct fast enough such that several ELM relaxations can be observed in a simulation, thus these simulations allow to give a qualitative comparison of the ELM frequency evolution with respect to parameters such as the heating power, but cannot give a quantitative comparison against experiment. Because of computational restrictions, the central resistivity is taken $\eta_{0}=2.5 \times 10^{-7} \Omega . \mathrm{m}$, which is 2 orders of magnitude larger than the Spitzer resistivity $\eta_{\text {Spitzer }}=2.5 \times 10^{-9} \Omega . \mathrm{m}$. The resistivity profile follows a $T^{-3 / 2}$ radial dependence. A JET

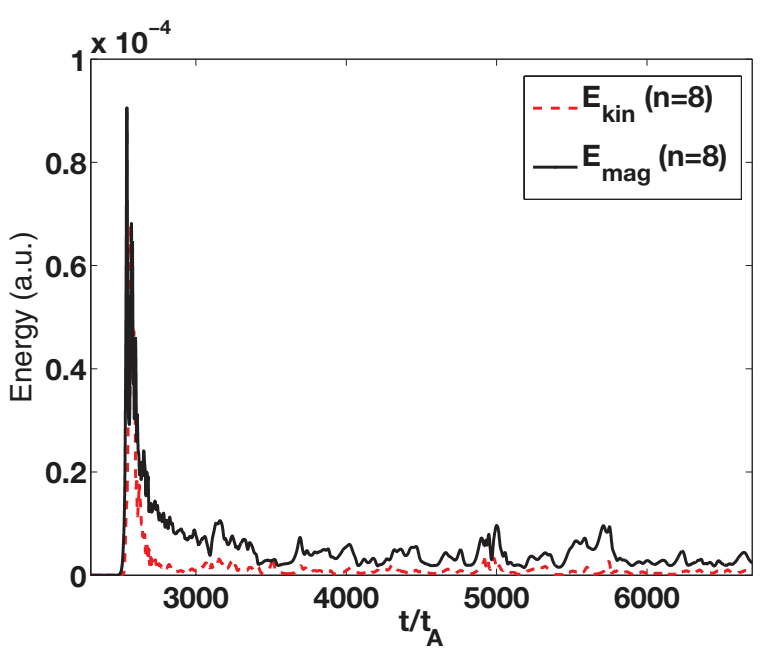

Figure 1. Time variation of the magnetic and kinetic energies of the mode $n=8$ without diamagnetic effects. Time is normalized to the Alfvén time $t_{A}$.

realistic diamagnetic velocity is taken for both species $s$ (ions and electrons): $\vec{V}_{s}^{*}=Z_{s}\left(R / R_{0}\right)^{2} \tau_{I C} / \rho \cdot \vec{b} \times \nabla P_{s} . Z_{s}$ and $P_{s}$ are respectively the charge number and the scalar pressure of the species $s, R$ is the horizontal coordinate, $\rho$ is the mass density and $\vec{b}$ is the magnetic field normalized to $B_{t}$. The realistic value of the diamagnetic parameter (inverse of the ion cyclotron frequency) $\tau_{I C}=m_{i} / e B_{t} \sim 7 \times 10^{-9} \mathrm{~s}$ is taken, and ion and electron temperatures are assumed to be equal. The model is the MHD model extended with bi-fluid diamagnetic flows, as described on [20]. Note that the neoclassical friction and the source of toroidal rotation are not included in this section in order to point out the role of diamagnetic stabilization.

The ELM crash is simulated first without diamagnetic rotation (section 2.1), then the same simulation is performed with diamagnetic drifts (section 2.2).

\section{1. simulations without diamagnetic drifts: single ELM crash}

In the modeling without diamagnetic drifts, the ELM crash is generated by the chosen initially unstable pressure profile. After the crash, the unstable modes remain unstable, and the residual magnetic activity expels the plasma outside the separatrix, which prevents the pedestal from building-up again. The example is given for the case of an $n=8$ unstable toroidal mode. The ELM crash corresponds to a large peak of magnetic and kinetic energy (figure 1). At the crash, the large magnetic activity leads to a strong ergodization of the edge (the magnetic field is ergodic for a normalized flux $\psi>0.85$, as shown in figure $2(a)$ ). After the crash, magnetic islands remain for $\psi>0.85$ and an ergodic layer subsists at the very edge $(\psi>0.95$, see figure $2(b))$. This magnetic activity and particularly the edge stochastization increase both the heat parallel diffusivity and the $E \times B$ convection of particles. The enhanced transport prevents the reconstruction of the pedestal profiles and keeps the plasma below the peeling-ballooning stability limit, thus a second ELM cannot be obtained in the simulations without diamagnetic effects. 


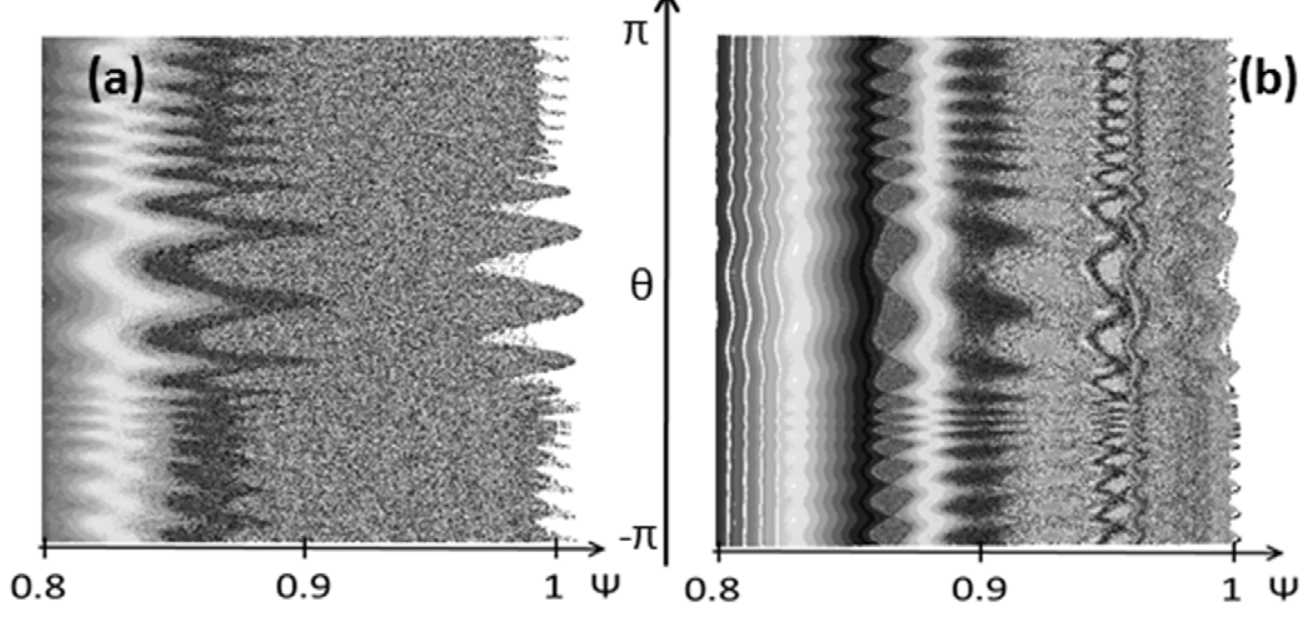

Figure 2. Poincaré plot of the magnetic topology at the plasma edge (normalized flux $\psi \geqslant 0.8$ ) in the $n=8$ ELM simulation without diamagnetic effect, given at the peak of the ELM crash $(a)$ and after the ELM crash at $t \sim 7 \times 10^{3} t_{A}(b)$. Note the full edge reconnection at the peak of the crash, and the remaining partial reconnection after the crash.

\subsection{Simulations with diamagnetic drifts: ELM cycling dynamics}

The diamagnetic effects are known to prevent the magnetic field from reconnecting and to reduce the growth rate of ideal and resistive instabilities [21, 22] and thus have a stabilizing effect on plasma instabilities. In particular they are evidenced to be a key parameter for simulating cycles of sawtooth crashes [23]. We show here that the diamagnetic drifts also enable to simulate cycles of ELM crashes. The diamagnetic stabilization has two major effects on ELMs. First, it reduces the amplitude of the ELM perturbation and crash. Indeed, compared to the simulation without diamagnetism (figure 1), the magnetic and kinetic energies of the same $n=8$ ELM with diamagnetic drifts included (figure 3 ) are 1-2 orders of magnitude lower. In figure 3 , the multi-harmonic simulation of the modes $n=0,2,4,6$ and 8 with diamagnetic drifts is presented. The most unstable $n=8$ mode is hardly coupled with the other modes during the first ELM crash and the energy of the other modes remain several orders of magnitude lower than the energy of the $n=8$ mode. So the dynamics of the first ELM is almost only governed by the $n=8$ mode. Note that the modes $n>9$ are stabilized by the diamagnetic stabilization; for this reason and in order to reduce the time-consumption of the simulation, the highest $n>9$ modes were not kept in simulation.

After the first ELM crash, we observe the second major role of the diamagnetic stabilization: instead of remaining unstable after the crash, the plasma is stabilized by the diamagnetic rotation (figure 3 ). Only when the pressure profile is built up by the applied heating power, the plasma is destabilized again. The ballooning modes are growing again until a threshold in pressure gradient is reached, triggering the second ELM relaxation. Therefore the ELM dynamics results from a competition between the diamagnetic stabilization and the destabilization of the plasma by the heat and particle sources filling the pedestal and steepening the pressure profile. The ELM cycles obtained in simulation (figure 3) can be

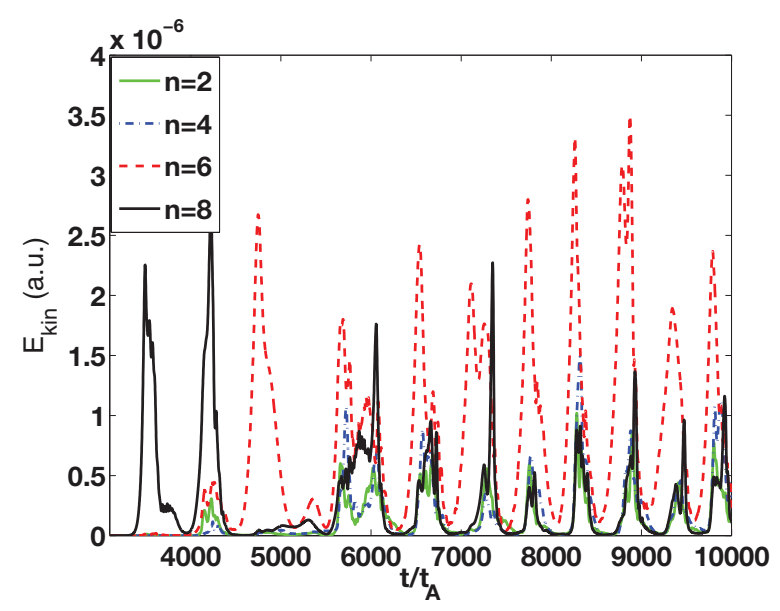

Figure 3. Kinetic energy of the modes $n=2-8$ in the multiharmonic $n=0: 2: 8$ simulation with diamagnetic effects.

decomposed into two periods. The first three transient ELMs are largely dominated by the most unstable modes $(n=8$ during the first two ELMs then $n=6$ ), and their triggering threshold is dependent on the initial chosen state. However, after 3-4 ELMs, the memory of the initial state is lost, and a quasi-periodic regime of ELM crashes is obtained. These ELMs are characterized by a strong non-linear coupling between the $n=2-8$ modes. The $n=6$ mode is growing first, directly followed by the other modes. After a crash in this quasi-periodic regime, the plasma reorganizes into a self-consistent state. Thus, the ELM frequency does not depend any more on initial conditions, but depends on intrinsic parameters: the diamagnetic rotation (which have a stabilizing effect on ELMs) and the applied heating power (destabilizing), as described in the next section 2.3. The competition between these two parameters governs the cyclical dynamics of the ELMs. Each ELM in the quasi-periodic regime can be decomposed into three steps (figure 4): first, the instability grows as the pressure gradient (and in a smaller extent the edge current) 

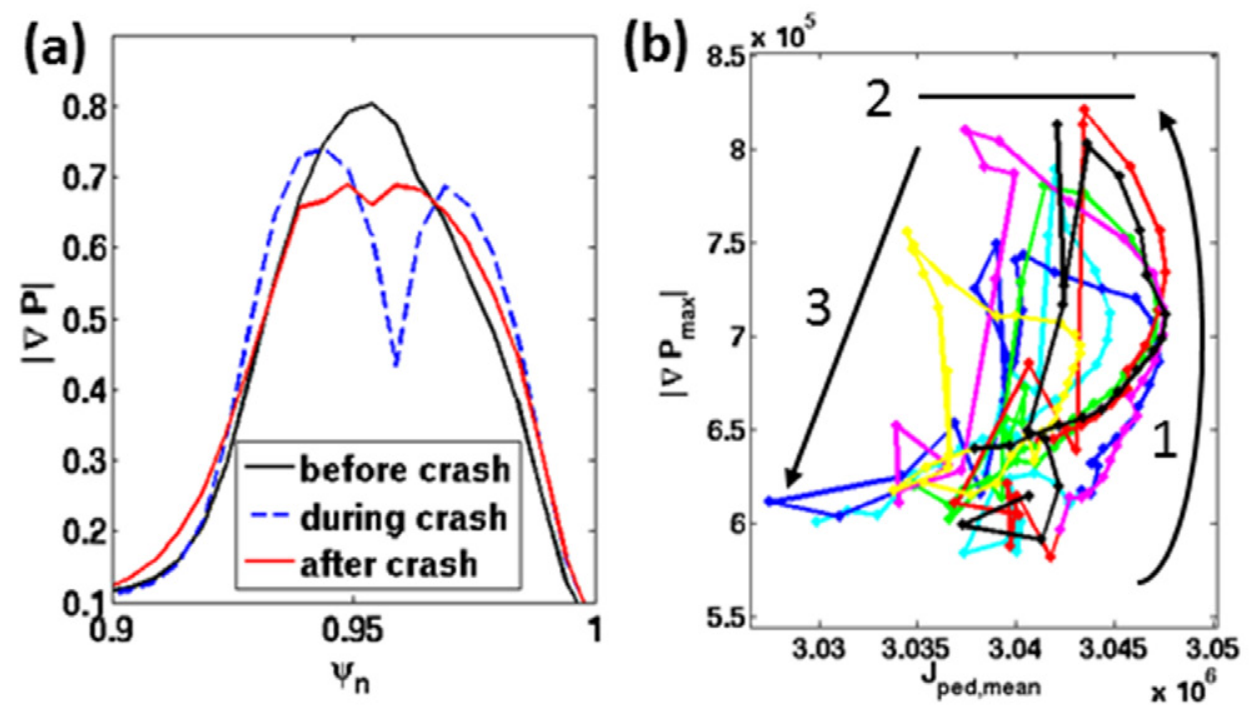

Figure 4. In quasi-periodic regime: (a) edge pressure gradient before, during and after an ELM. (b) Peeling-ballooning diagram of the ELMs.
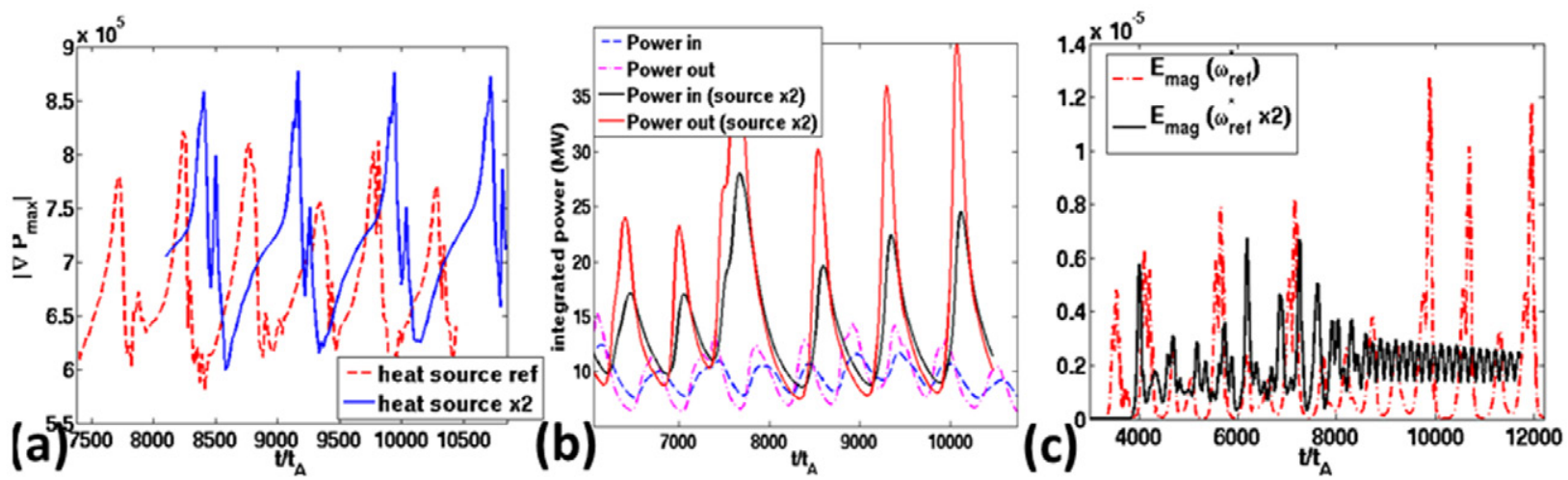

Figure 5. $(a)-(b)$ Time variation of the maximal edge pressure gradient $(a)$ and integrated power on the inner and outer divertor plates $(b)$ in quasi-periodic $n=8$ ELMy regime in the reference case (dash) and with a double injected power. (c) Magnetic energy of the $n=8$ ELM depending on the diamagnetic parameter: realistic value (dash) and doubled (full).

increase. Second, the ELM crash occurs when a limit of pressure gradient is reached. And third, the pedestal profiles relax: particles and energy are expelled out of the plasma, and the pressure gradient collapses, as presented in figure 4(a). Note that the bootstrap current is not included in the model (the current is forced to tend to the initial realistic current profile via a current source, but does not depend on the pressure gradient evolution, as it is in experiments), so the dynamics of the instabilities simulated here shows only a small dependence on the pedestal current (figure $4(b)$ ).

\subsection{Characterization of the ELM frequency}

The ELM frequency obtained in figure 3 is about $3 \mathrm{kHz}$. This frequency cannot be compared quantitatively with experiments, due to the ad hoc sources taken in simulations; however a qualitative study on the parameters impacting the ELM frequency can be done. The ELM frequency depends on both the stabilizing effect of the diamagnetic rotation and the destabilization by the steepening of the pressure profile due to the heat source. To assess these effects, simulations are performed first with a twice larger heat source and second with a twice larger diamagnetic parameter $\tau_{I C}$. First, we notice that the enhanced heat source has the effect of raising the maximal pressure gradient reached at the ELM crash, which is about $5 \%$ larger with the enhanced source (figure $5(a)$ ). This can be explained by two combined effects. First, whilst the peelingballooning modes are growing, the enhanced heating power makes that the pressure gradient rise more rapidly during the delay needed by the non-linear effects to induce the pedestal relaxation. Second, due to the larger heating power, the mean equilibrium (inter-ELM) temperature $T_{0}$ is raised after several ELMs. Consequently, the resistivity (proportional to $T_{0}^{-3 / 2}$ ) is reduced, so the boundary stability is increased. For both reasons, a larger pressure gradient develops in the pedestal before the ELM crash, which induces a larger amount of particles and energy expelled by the ELM. Subsequently, the power reaching the divertor (figure $5(b)$ ) is increased. This means that the pedestal has emptied more consequently and that more time is needed to reconstruct the pedestal. Therefore the ELM frequency is decreased as the heat power is increased. This behavior is closer to the type-III ELMs obtained in the 

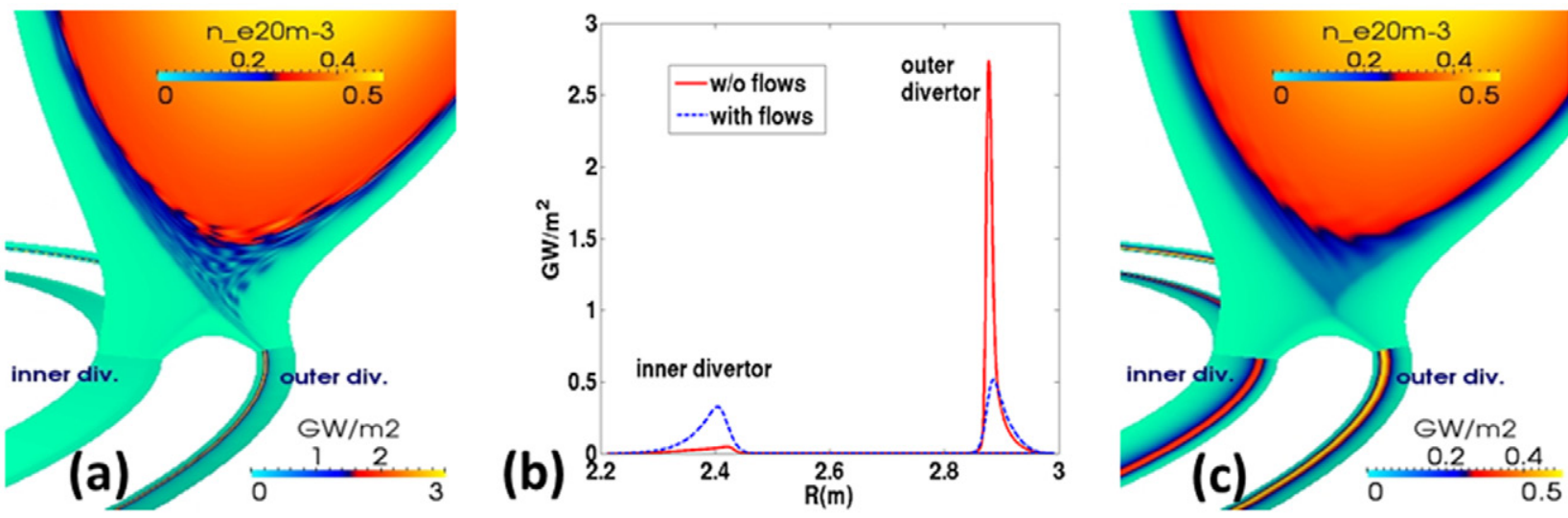

Figure 6. Heat flux (in $M W / \mathrm{m}^{2}$ ) reaching the inner and outer divertor target plates after an ELM: without flows ( $a$ ) or with diamagnetic, neoclassical and toroidal flows $(c)$. A radial section along the divertor is plotted in $(b)$ : almost all flux reaches the outer divertor (full line) without flows whereas the deposition is near-symmetric with flows (dash line).

experiments [24]. Yet the type-III ELM instabilities are so far not clearly understood theoretically, and since both the type-I and type-III ELMs are linked with the same instabilities (peeling-ballooning), it is still not clear what fundamentally differentiates these two types. More modeling with realistic parameters (allowing for a close comparison to experiments) is planned to better characterize the difference between type-I and type-III ELMs.

Second, simulations at larger diamagnetic rotation present a larger ELM frequency. Actually, due to the diamagnetic stabilization, smaller instabilities develop at the edge plasma and a smaller energy is expelled from the pedestal. So the plasma after the crash remains just below the stability limit, such that less time is needed to cross again the stability threshold, which explains a larger ELM frequency. In the case with a diamagnetic parameter twice larger than the realistic one (figure 5(c)), not only the ELM frequency (obtained after the transient phase) is increased, but the large diamagnetic rotation gradually decreases the amplitude of the ELMs, so that in terms of peeling-ballooning cycles, the plasma moves from the initially unstable state towards a 'fix point' in the P-B diagram by doing smaller and smaller cycles. As it represents a bifurcation from a cycling dynamics towards a stable state, it may present similarities with the so-called dithering cycles [4].

\subsection{Near-symmetric power deposition on divertor target plates}

The diamagnetic rotation also affects the dynamics of the ELM precursors and filaments. Without diamagnetic rotation, the instabilities at the onset of the ELM have a static growth, whereas the diamagnetic rotation makes the ELM precursors grow and rotate in the electron diamagnetic direction at the speed $V_{\text {mode }} \approx V_{E \times B}+V_{i}^{*} / 2$ [25]. In the non-linear stage, the plasma inside the separatrix rotates in the electron diamagnetic direction, and the ELM filaments are sheared out of the plasma in the ion diamagnetic direction. The temperature is conducted by the heat diffusivity from the reconnected edge plasma to the divertor plates, whereas the density is convected by the $E \times B$ and diamagnetic rotation. It is important to notice that this diamagnetic advection of the density makes that more density reaches the inner divertor plate than the outer plate, hence the heat flux reaching the divertor at the sound speed is deposited near-symmetrically in the inner and outer divertor plates: even though the temperature is larger on the outer than on the inner side, the density is larger in the inner region so a similar power, proportional to both the plasma temperature and density, reaches the inner and outer divertor target plates due to an ELM. As an example, figures $6(b)$ and $(c)$ present the near-symmetric power deposition of the heat flux in the simulation of a realistic JET shot (\#77329, described in [26]) where the diamagnetic, neoclassical and toroidal flows have been taken into account. In comparison, in simulations made without flows (figures $6(a)$ and $(b)$ ), the outer divertor received almost all the heat power. This was in contradiction with the experimental observations where the deposit is either symmetric on inner and outer divertor plates or two times larger in the inner divertor $[27,28]$. Thus simulations with diamagnetic drifts allow for a more realistic reproduction of the ELM dynamics up to the deposition on the divertor.

\section{ELM control by RMPs}

The plasma rotation and in particular the diamagnetic rotation is also known to affect the penetration of the RMPs in the plasma $[20,26,29,30]$. Previous studies demonstrated that the plasma perpendicular $(E \times B+$ diamagnetic) rotation is likely to prevent the RMPs from penetrating into the plasma. Thus RMPs can just penetrate on particular rational surfaces where the perpendicular rotation is zero and at the very edge where the resistivity is the highest. In [20], we found that even though RMPs are screened in the bulk plasma, they still affect the magnetic topology: small islands are generated on the rational surfaces $q=m / n$ and an ergodic layer is formed at the very edge, which induces an enhanced transport at the edge. In this section, we find that the perpendicular diamagnetic rotation also plays an important role in the interaction between ELMs and RMPs in the plasma. 


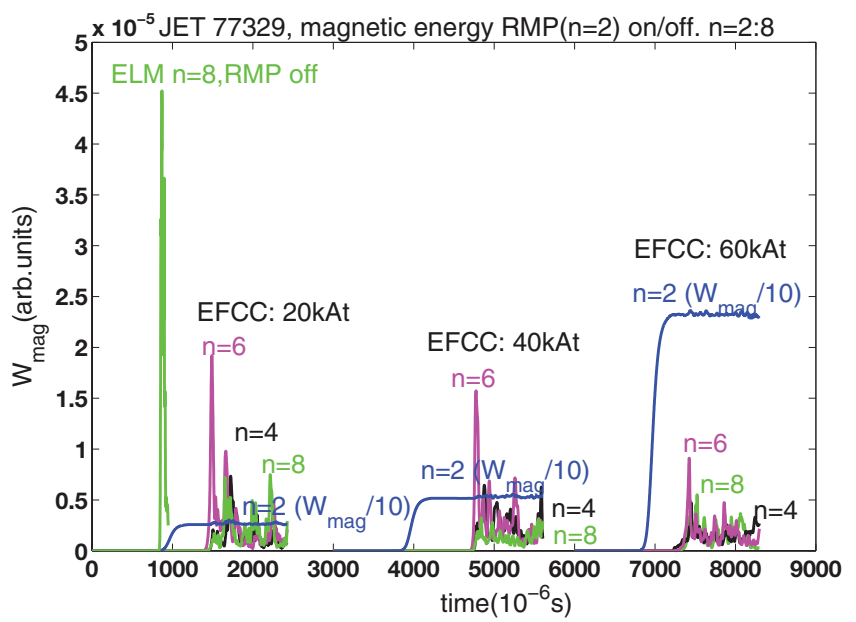

Figure 7. Magnetic energy of the modes $n=2-8$; from left to right: without RMP, with RMP current $I_{\text {coil }}=20 \mathrm{kAt}$, 40kAt and $60 \mathrm{kAt}$.

\subsection{ELM power deposition divided by ten with RMPs}

In this section, experimental JET parameters corresponding to the shot \#77329 [26] are used. Typical JET values are taken for the diamagnetic parameter and the neoclassical coefficients as in $[20,26]$. The $(n=2)$ RMP spectrum due to the error field correction coils (EFCC) is first calculated in the vacuum with the ERGOS code ([31]) and applied as boundary conditions for the magnetic flux perturbation in JOREK. Once the plasma response to $(n=2)$ RMPs has stabilized to an equilibrium (affected by RMPs), the other toroidal modes are added to the simulation. The effect of RMPs on ELMs is tested first in a simulation with $n=0,2,4,6$ and 8, the RMP coil current being scanned between 20 and $60 \mathrm{kAt}$. In the simulation run without RMPs, the most unstable mode is the $n=8$, which leads to a large ELM crash (figure 7, left). As compared to the ELM cycle considered in section 2, where the ELMs induced rather small pedestal relaxations (the integrated peak power reaching the inner and outer divertor targets was about 10-30 MW depending on the applied heating power, as shown in figure $5(b)$ ), the ELM considered in this part induces a much larger relaxation (around $100 \mathrm{MW}$ of integrated power reach each divertor target, as plotted in figure 8) when unmitigated. This large ELM is thus rather related to large type-I ELMs observed in experiments, yet further modeling is foreseen to compare more quantitatively this ELM with experimental observations. When RMPs are applied, instead of this large ELM crash, a more continuous activity of the modes $n=2,4,6$ and 8 coupled altogether is observed (figure 7). This activity starts with a peak of energy of the mode $n=6$, which is the most unstable with RMPs. This peak is smaller compared to the peak of the $n=8$ mode without RMPs thus it leads to a mitigated ELM with a smaller peak energy released. Moreover, as the RMP current is increased from 20 to $60 \mathrm{kAt}$, the $n=6$ peak size is reduced, which means that a stronger ELM mitigation is obtained when the RMP current is larger. The power reaching the divertor in the $40 \mathrm{kAt}$ case is plotted in figure 8. The deposited power is divided by ten when RMPs are applied compared to the 'natural' ELM (without RMPs).

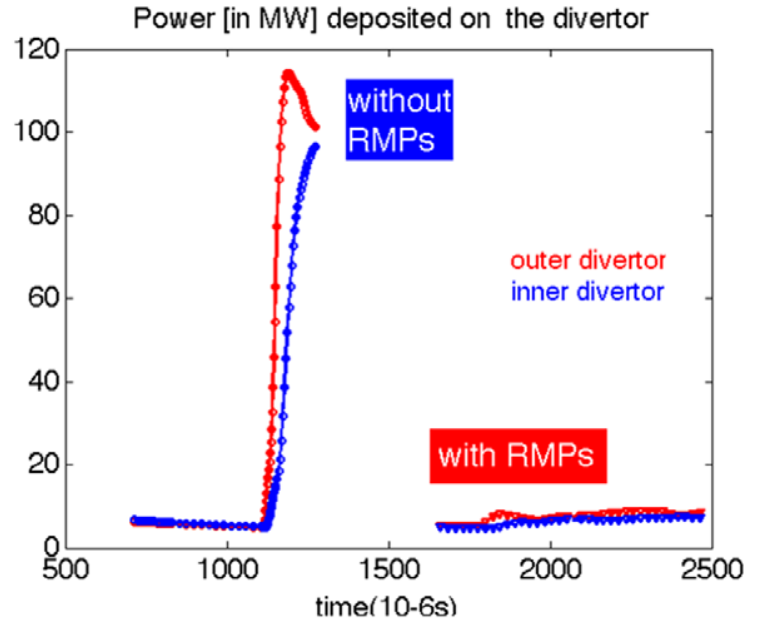

Figure 8. Power on inner/outer divertor targets without (left) and with RMPs (right) for $I_{\text {coil }}=40 \mathrm{kAt}$.

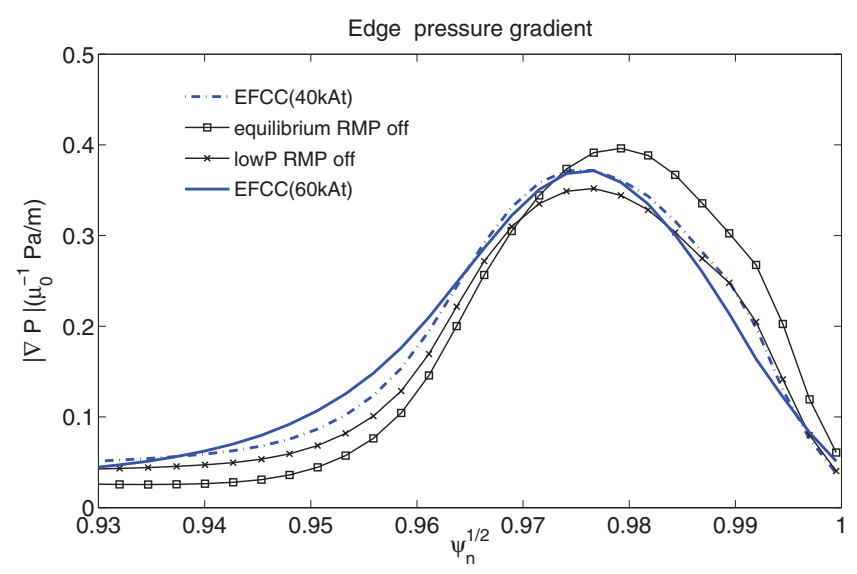

Figure 9. Edge pressure gradient: cases without RMP (squares), with RMP at $I_{\text {coil }}=40 k A t$ (dash-dot) and $60 \mathrm{kAt}$ (full line) and without RMP at reduced pressure gradient (crosses).

\subsection{Mechanism of the ELM mitigation by RMPs}

We try to understand the mechanism at stake in the ELM mitigation. The first hypothesis is that the edge ergodization due to RMPs which results in a lower edge pressure gradient (as shown in figure 9) may generate a (partial) stabilization of the ELMs. To test this hypothesis, a simulation is run without RMPs with a reduced pressure gradient similar to the pressure gradient obtained with RMPs (figure 9, line with crosses). In fact, the reduction of the edge pressure gradient delays the ELM crash (full line in figure 10) compared to the reference ELM (dashed line in figure 10), but still leads to a large ELM crash. So the only reduction of the pressure gradient by RMPs does not explain the observed ELM mitigation. The second tested hypothesis in that the modification of the magnetic topology due to RMPs induces the mitigation. A linear run of an ELM with RMPs is thus performed, where the magnetic topology is affected by both the ELM and the RMPs but where the RMPs and the unstable modes are not coupled. This linear run also leads to a large ELM crash. 


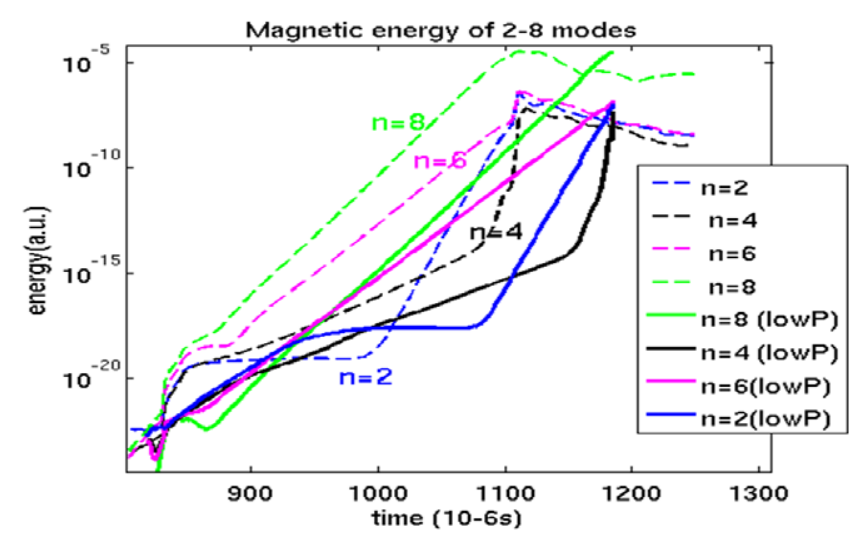

Figure 10. Magnetic energy of the modes $n=2: 8$ without RMP: in the simulation run at reduced pressure gradient (full line), the ELM crash is delayed compared to the reference case, but remains large.

This proves that it is really the coupling between the $(n=2)$ RMPs and its harmonics (even modes) that generate the ELM mitigation. Figure 11 presents the simulation at $60 \mathrm{kAt}$ with $n=0-8$. We notice that the initial growth rate of the even modes $n=4,6$ and 8 is large due to the non-linear drive by $(n=2)$ RMPs. The magnetic energy between these even modes is equally redistributed after the first relaxation of the $n=6$ modes and the magnetic energy non-linearly cascades from the naturally unstable $n=8$ mode towards the lower $n$ even modes [32]. As for the odd modes (figure 11), they are totally damped due to the activity of the even modes driven by RMPs, and hence they remain at the noise level. The reduction of the ELM toroidal mode number when mitigated by RMPs was observed in KSTAR [33], but not in the general case [34]. Actually, in another simulation performed at $15 \%$ larger diamagnetic rotation (not presented here), the $n=6$ mode is the most unstable for the natural ELM due to the diamagnetic stabilization of the larger $n$ modes, and remains the most unstable mode while non-linearly coupled with $(n=2)$ RMPs. Thus the energy cascade implies a redistribution between the non-linearly coupled modes but does not necessarily mean a reduction of the main toroidal mode number.

The coupling of ELM and RMPs induce a change in the edge magnetic structure, as plotted in figure 13. The magnetic topology of the natural ELM (figure 13(a)) is dominated by the $n=8$ ballooning perturbation that induces a large reconnection at the edge (for $\psi_{\text {norm }}>0.85$ ). In the case of RMPs without ELMs (figure 13(b)), $n=2$ magnetic islands are formed on the rational surfaces $q=m / n$ due to the forced magnetic reconnection, and present a tearing-like parity $\left(\psi_{m n} \neq\right.$ 0 on the rational surfaces). It is important to notice that the perpendicular $(E \times B+$ diamagnetic) electron rotation is zero on the rational surface $q=5 / 2$ (at $\psi_{\text {norm }} \approx 0.85$ ) which leads to the RMP penetration and the formation of large islands on this surface, as demonstrated in [20, 26, 29]. As for the magnetic topology of the ELMs with RMPs (figure 13(c)), it is dominated by the modes $n=4$ and $n=6$, with magnetic islands observed on the rational surfaces $q=9 / 4,14 / 6$ and $15 / 6$. The overlapping of the surfaces $q=16 / 6,17 / 6$ and $18 / 6$ generates an ergodic zone deeper in the pedestal than in the

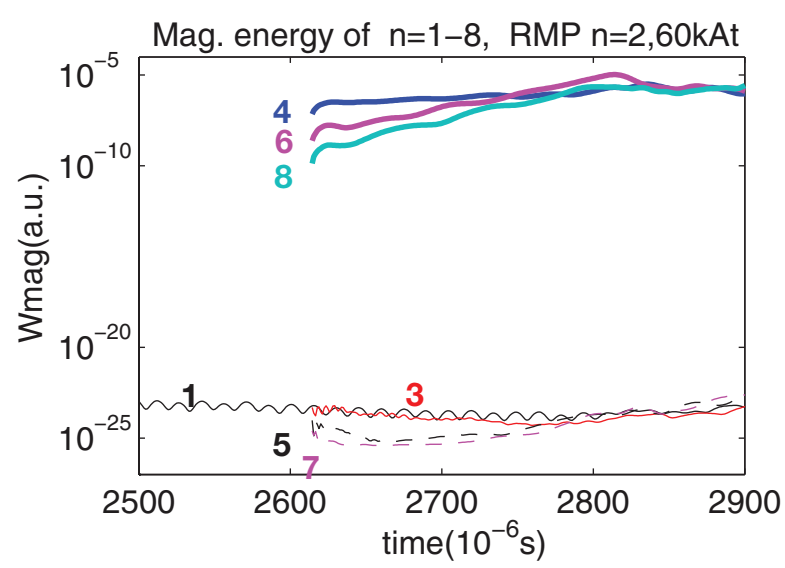

Figure 11. Magnetic energy of the modes $n=1-8$ with RMPs $\left(I_{\text {coil }}=60 \mathrm{kAt}\right)$. The magnetic activity due to the even modes driven by RMPs totally damps the odd modes.

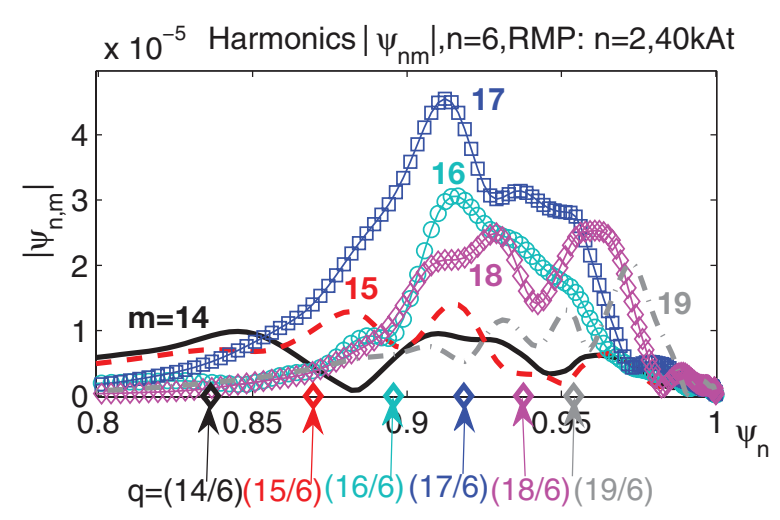

Figure 12. Radial profiles of the Fourier harmonics of the magnetic flux perturbation $\left|\psi_{m n}\right|$, for $n=6$ and $14<m<19$. The rational surfaces $q=m / n$ are indicated by the diamonds.

case of RMPs alone. The $n=4$ and 6 modes have a tearingparity $\left(\psi_{m n} \neq 0\right)$ on these rational surfaces that is imposed by the driving by RMPs (as plotted in figure 12), in place of the ballooning parity in the natural ELM case $\left(\psi_{m n}=0\right)$. The magnetic islands and above all the ergodic zone at the edge induce an enhanced transport at the edge that expels continuously the heat and particles out of the plasma. Note that no clear density pump-out is observed in our simulations of ELMs mitigated by RMPs: the density is only affected in the pedestal and less than $1 \%$ of the total plasma density is lost due to the RMP application. Actually two groups of RMP experiments can be distinguished. At high collisionality, the RMP application usually leads to the replacement of large ELMs by small frequent ELMs or MHD turbulence, and the heat flux on the divertor is reduced by RMPs. Small changes in the edge pressure gradient and MHD stability are reported $[8-12,35]$. On the other hand, at low collisionality resulting from the strong density pump-out induced by RMPs, ELMs have been totally suppressed in DIII-D and the plasma edge appears to be stable to peeling/ballooning modes [7]. These simulations thus reproduce quite accurately the main features of the ELM mitigation at high collisionality. A study is under way to be able to also model the pumpout observed at low 


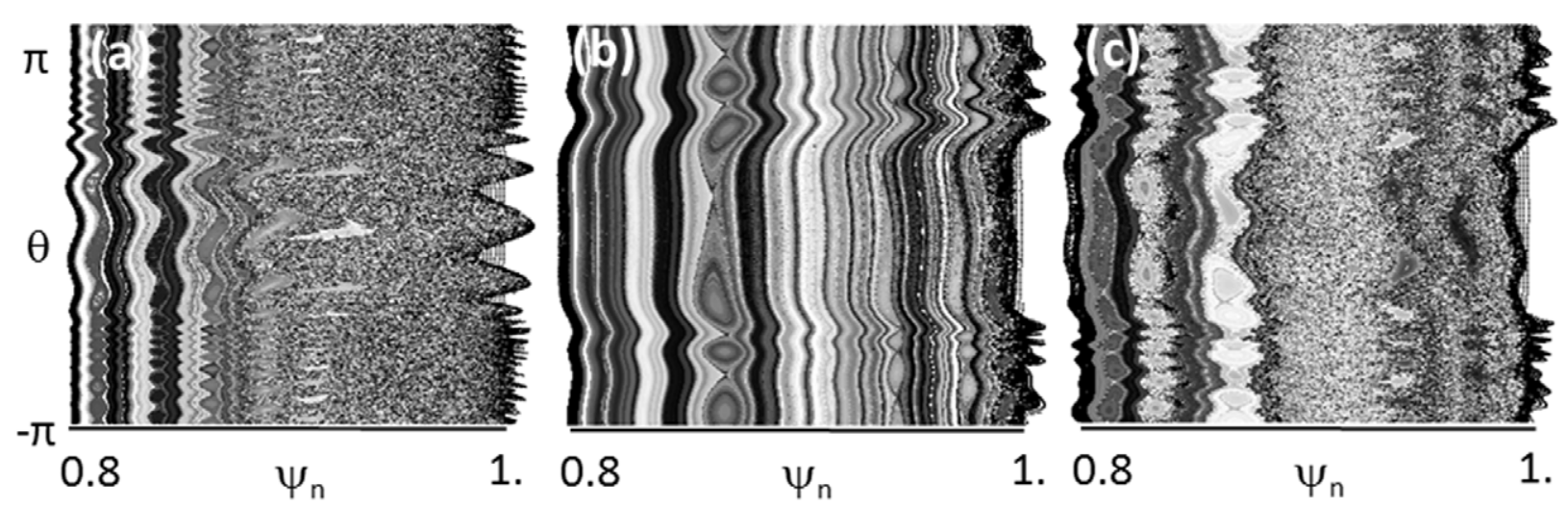

Figure 13. Poincaré plot of the magnetic topology at the edge $\left(0.8<\psi_{\text {cnorm }}<1\right)$ : in case of an ELM without RMP $(a)$, in case of RMPs without $\operatorname{ELM}(b)$ and in case of an ELM with RMPs $(c)$.

collisionality. Reference [36] demonstrated that the parallel current flowing along perturbed magnetic field lines (phenomenon called 'magnetic flutter') generates an enhanced radial transport of the electron density, which may be responsible for the pump-out of density observed in RMP experiments at low collisionality. In this respect, the parallel current flow has been added into the electron density equation in the JOREK model so as to test the effect of the magnetic flutter on the density transport, and thus observe the density pump-out in modeling. This aspect will be raised in future works.

It is also important to note that depending on the q profile taken (in experiments as well as in simulations), the penetration of the RMPs and thus their effect on ELMs may be different. As an example, the ELM suppression was obtained for a narrow $q_{95}$ 'resonant window' in DIII-D [7]. This narrow resonant window is believed to be linked with the presence of a rational surface at the top of the pedestal where the perpendicular electron velocity vanishes [26, 29], inducing the penetration of the RMPs (i.e. the formation of an island chain) on this surface. When the $q_{95}$ parameter is varied, the resonant surface is shifted towards a position where the perpendicular electron flow is larger and thus screens the RMPs. This might explain the presence of a $q_{95}$ window for which RMPs penetrate and thus mitigate or suppress the ELMs. However the ELM mitigation was observed for a wide $q_{95}$ window in JET [37] and Asdex Upgrade [38], so the importance of this 'resonance effect' is not clear. In our simulations, the presence of the island chain induced by RMPs at the position where the perpendicular electron velocity is close to zero (at $\psi_{\text {norm }} \approx 0.85$ ) might play an important role on the ELM mitigation, yet the region of importance where the ergodic layer is formed is located closer to the edge (around $\psi_{\text {norm }}=0.9$ ). More modeling for different $q_{95}$ values is planned to check if a resonance effect is found.

\subsection{ELM cycle with RMPs}

A multi-harmonic ( $n=2,4,6$ and 8) simulation of an ELM cycle with $(n=2)$ RMPs is performed for a larger diamagnetic rotation (this is done both by increasing the diamagnetic parameter $\tau_{I C}$ and by taking a steeper pressure profile). In this case, the most unstable mode without RMPs is the $n=6$ mode (in red in figure 14) due to the diamagnetic stabilization of the larger mode numbers. At rather low applied RMP current (40kAt, left of figure 14), the ELMs are not mitigated by RMPs. Neither the ELM amplitude or frequency is affected by the RMPs. This may be explained by the large screening of RMPs at large diamagnetic (perpendicular) rotation, which does not allow for a large coupling between unstable modes and RMPs. However at twice larger applied RMP current (80kAt, right of figure 14), the modes $n=4,6$ and 8 are more strongly coupled to RMPs, thus they are now fully driven by $n=2$ RMPs and present a large initial amplitude due to the coupling with RMPs. Contrary to the case presented in section 3.1, these coupled modes do not generate a turbulent MHD activity. Instead, static islands driven by RMPs are formed. The presence of these islands-reconnected at the edge-generates a permanently enhanced heat and particle transport, which reduces the edge pressure gradient and thus keeps the plasma under the stability threshold. Therefore, by increasing the RMP current from 40 to $80 \mathrm{kAt}$, a bifurcation from a largely screened state toward a reconnected state takes place [39], which leads to the full ELM suppression. Subsequently we have observed three different regimes: ELM mitigation by RMPs, no effect of RMPs on ELMs and ELM suppression by RMPs. These regimes depend on both the RMP amplitude and the diamagnetic rotation. Future work will focus on quantifying more precisely the access to these different regimes.

\section{Discussion and conclusion}

The ELM cyclical dynamics as well as the ELM mitigation by RMPs was observed in non-linear modeling performed with the extended MHD code JOREK. In both cases, the diamagnetic rotation was found to be a key parameter. The main limitations of our model is the large resistivity which is two orders of magnitude larger than the experimental value due to computational limitations and the ad hoc heat and particle sources taken in simulation that affect the ELM frequency. In the simulation of ELM cycles, the diamagnetic stabilization allows for suppressing the MHD activity after the crash, and 

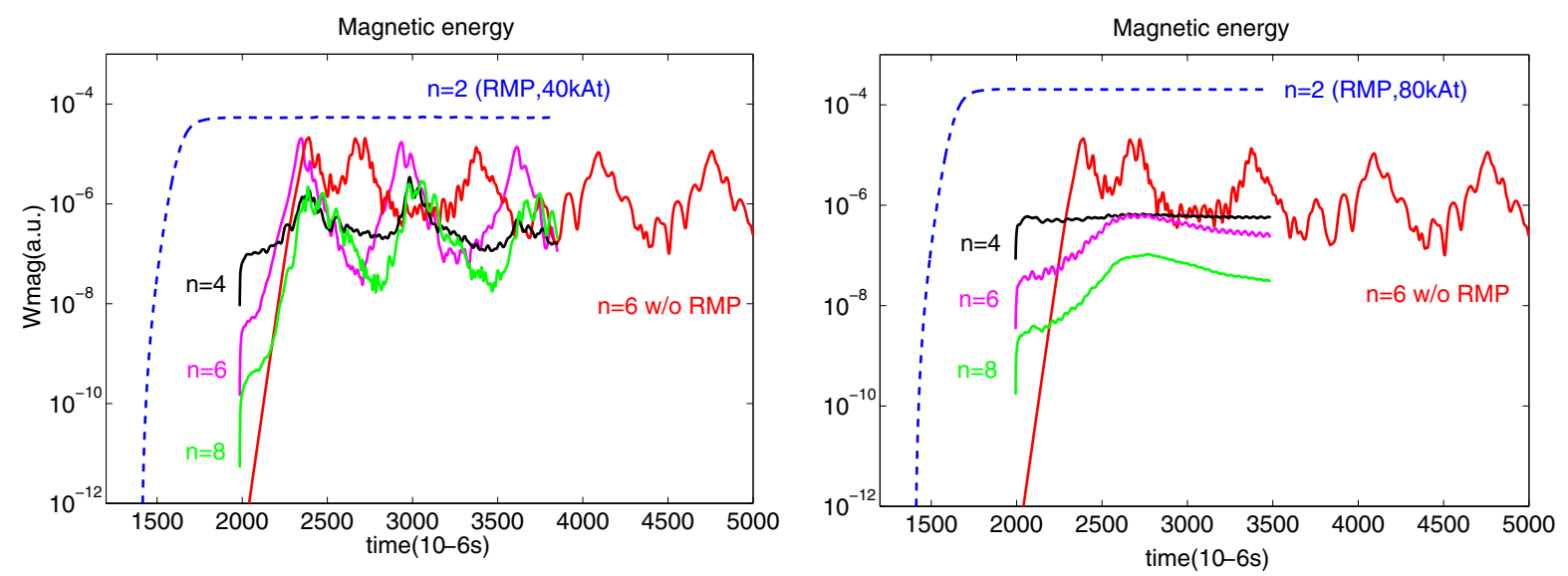

Figure 14. Comparison of the magnetic energy of the $n=6$ mode without and with RMPs (in log scale). At 'low' RMP current (40kAt, left), RMPs have no effect on the ELM amplitude and frequency, whereas at larger RMP current (80kAt, right), the ELM is fully stabilized by RMPs.

a competition between the diamagnetic stabilization and the destabilization by the steepening of the pressure profile-due to the applied heating power-results in a cyclical dynamics. If the diamagnetic rotation is largely increased, the ELM frequency is increased and the ELM size is progressively reduced, so that the plasma gravitates towards an attracting point in the peeling-ballooning diagram. In our particular case of resistive (type-III like) ELMs, an enhanced heat source leads to a larger maximal pressure gradient reached at the ELM crash, resulting in a larger ELM frequency and a larger heat flux reaching the divertor. Concerning the deposition on the divertor, the diamagnetic drifts are found to convect the density towards the inner plate, which generates a nearsymmetric deposition on the inner and outer divertor plates: this is closer to the experimental results compared to previous simulations without diamagnetic rotation.

As for the ELM control by RMPs, three regimes have been found in the JET case with $n=2$ RMPs. First, at rather low diamagnetic rotation, the ELM mitigation is obtained, where the large ELM crash is replaced by a continuous (in time) magnetic activity or turbulence, also called type-II ELMy activity [7-9]. The size of the small initial relaxation is reduced as the RMP coil current is increased. For $I_{\text {coil }}=40 \mathrm{kAt}$, the integrated peak power on the divertor is found to be divided by a factor of ten. The ELM mitigation is not due to the reduction of the edge pressure gradient but is rather due to the non-linear coupling of the even modes which are driven by the $n=2$ RMPs. These RMP-driven modes generate plasma reconnectioncharacterized by magnetic island chains in the pedestal and an edge ergodic zone-and thus continuously expel heat and particles out of the plasma. Then, at larger diamagnetic rotation, two other regimes are observed in simulation. At rather low RMP current $\left(I_{\text {coil }}=40 k A t\right)$, the RMPs have no effect on the ELM dynamics: the ELM amplitude and frequency are not affected by the RMPs since the RMPs are too 'shielded' by the plasma rotation to be coupled with unstable modes. However, at larger RMP current $\left(I_{\text {coil }}=80 k A t\right)$, RMPs penetrate and are strongly coupled with the even modes, such that these modes are 'locked' to the RMP driving: hence the large permanent transport induced by these RMP-driven modes stabilizes the plasma under the stability threshold and the ELMs are fully suppressed. Current and future works are dedicated to a more precise understanding of the parameters that demarcate these three regimes.

\section{Acknowledgments}

This work has been carried out within the framework of the EUROfusion Consortium. It has received funding from the European Union Horizon 2020 research and innovation programme under grant agreement number 633053 and from the National French Research Program (ANR): ANEMOS (2011) and E2T2 (2010). This work was granted access to the HPC resources of Aix-Marseille Université financed by the project Equip@Meso (ANR-10-EQPX-29-01). A part of this work was carried out using the HELIOS supercomputer system (IFERC-CSC), Aomori, Japan, under the Broader Approach collaboration, implemented by Fusion for Energy and JAEA, and using the CURIE supercomputer, operated into the TGCC by CEA, France, in the framework of GENCI and PRACE projects. The views and opinions expressed herein do not necessarily reflect those of the European Commission or the ITER Organization.

\section{References}

[1] Snyder P B, Wilson H R, Osborne T H and Leonard A W 2004 Characterization of peeling-ballooning stability limits on the pedestal Plasma Phys. Control. Fusion 46 A 131

[2] Kirk A et al 2014 Recent progress in understanding of the processes underlying the triggering of and energy loss associated with type I ELMs Nucl. Fusion accepted

[3] Dunai D et al 2014 Measurements of type I ELM triggering mechanism in MAST and KSTAR Proc. of the 41st EPS Conf. (Berlin, 23-27 June 2014)

[4] Zohm H 1996 Edge localized modes (ELMS) Plasma Phys. Control. Fusion 38105

[5] Hawryluk R J et al 2009 Principal physics developments evaluated in the ITER design review Nucl. Fusion 49065012 
[6] Fenstermacher M E et al 2008 Effect of island overlap on edge localized mode suppression by resonant magnetic perturbations in DIII-D Phys. Plasmas 15056122

[7] Evans T E et al 2008 RMP ELM suppression in DIII-D plasmas with ITER similar shapes and collisionalities Nucl. Fusion 48024002

[8] Jeon Y M et al 2012 Suppression of edge localized modes in high-confinement KSTAR plasmas by nonaxisymmetric magnetic perturbations Phys. Rev. Lett. 109035004

[9] Suttrop W et al 2011 First observation of edge localized modes mitigation with resonant and nonresonant magnetic perturbations in ASDEX Upgrade Phys. Rev. Lett. 106225004

[10] Liang Y et al 2007 Active control of type-I edge-localized modes with $n=1$ perturbation fields in the JET tokamak Phys. Rev. Lett. 98265004

[11] Kirk A et al 2010 Resonant magnetic perturbation experiments on MAST using external and internal coils for ELM control Nucl. Fusion $\mathbf{5 0} 034008$

[12] Canik J M et al 2010 ELM destabilization by externally applied non-axisymmetric magnetic perturbations in nstx Nucl. Fusion $\mathbf{5 0} 034012$

[13] Sugiyama L E and Strauss H R 2010 Magnetic x-points, edge localized modes, and stochasticitya Phys. Plasmas $\mathbf{1 7} 062505$

[14] Xu X Q et al 2011 Nonlinear ELM simulations based on a nonideal peeling-ballooning model using the bout ++ code Nucl. Fusion 51103040

[15] Xi W, Xu P Q and Diamond P H 2014 Phase dynamics criterion for fast relaxation of high-confinement-mode plasmas Phys. Rev. Lett. 112085001

[16] Sovinec C R et al 2007 Two-fluid studies of edge relaxation events in tokamaks J. Phys.: Conf. Ser. 78012070

[17] Huysmans G T A, Pamela S, van der Plas E and Ramet P 2009 Non-linear MHD simulations of edge localized modes (ELMS) Plasma Phys. Control. Fusion 51124012

[18] Krebs I, Hoelzl M, Lackner K and Guenter S 2013 Nonlinear excitation of low-n harmonics in reduced magnetohydrodynamic simulations of edge-localized modes Phys. Plasmas 20082506

[19] Pamela S J P et al 2013 Resistive mhd simulation of edgelocalized-modes for double-null discharges in the mast device Plasma Phys. Control. Fusion 55095001

[20] Orain F et al 2013 Non-linear magnetohydrodynamic modeling of plasma response to resonant magnetic perturbations Phys. Plasmas 20102510

[21] Drake J F, Antonsen T M, Hassam A B and Gladd N T 1983 Stabilization of the tearing mode in high-temperature plasma Phys. Fluids 262509

[22] Huysmans G T A, Sharapov S E, Mikhailovskii A B and Kerner W 2001 Modeling of diamagnetic stabilization of ideal magnetohydrodynamic instabilities associated with the transport barrier Phys. Plasmas 8 4292-305
[23] Halpern F D, Ltjens H and Luciani J-F 2011 Diamagnetic thresholds for sawtooth cycling in tokamak plasmas Phys. Plasmas 18102501

[24] Sartori R et al 2004 Study of type-III elms in JET Plasma Phys. Control. Fusion 46723

[25] Morales J A et al 2014 Rotation of edge localized modes and Their filaments Proc. of the 41st EPS Conf. (Berlin, 23-27 June 2014)

[26] Bécoulet $\mathrm{M}$ et al 2012 Screening of resonant magnetic perturbations by flows in tokamaks Nuc. Fus. 52054003 (5th Int. Workshop on Stochasticity in Fusion Plasmas (Julich, Germany, 11-14 April 2011))

[27] Pitts R A et al 2007 ELM transport in the JET scrape-off layer Nucl. Fusion 471437

[28] Eich T, Herrmann A and Neuhauser J 2003 Nonaxisymmetric energy deposition pattern on ASDEX Upgrade divertor target plates during type-I edge-localized modes Phys. Rev. Lett. 91195003

[29] Nardon E, Tamain P, Bécoulet M, Huysmans G and Waelbroeck F L 2010 Quasi-linear MHD modelling of $\mathrm{H}$-mode plasma response to resonant magnetic perturbations Nucl. Fusion $\mathbf{5 0} 034002$

[30] Ferraro N M 2012 Calculations of two-fluid linear response to non-axisymmetric fields in tokamaks Phys. Plasmas 19056105

[31] Bécoulet $\mathrm{M}$ et al 2008 Numerical study of the resonant magnetic perturbations for type-I edge localized modes control in iter Nucl. Fusion $\mathbf{4 8} 024003$

[32] Biskamp D 1997 Nonlinear Magnetohydrodynamics vol 1 (Cambridge: Cambridge University Press)

[33] Yun G S et al 2011 2D visualization of growth and burst of the edge-localized filaments in KSTAR H-mode plasmas Phys. Rev. Lett. 107045004

[34] Kirk A et al 2013 Effect of resonant magnetic perturbations with toroidal mode numbers of 4 and 6 on edge-localized modes in single null $\mathrm{H}$-mode plasmas in MAST Plasma Phys. Control. Fusion 55015006

[35] Evans T E et al 2004 Suppression of large edge-localized modes in high-confinement DIII-D plasmas with a stochastic magnetic boundary Phys. Rev. Lett. 92235003

[36] Waelbroeck F L, Joseph I, Nardon E, Bécoulet M and Fitzpatrick R 2012 Role of singular layers in the plasma response to resonant magnetic perturbations Nucl. Fusion 52074004

[37] Liang Y et al 2007 Active control of type-I edge localized modes on JET Plasma Phys. Control. Fusion 49 B581

[38] Suttrop W et al 2011 Studies of edge localized mode mitigation with new active in-vessel saddle coils in asdex upgrade Plasma Phys. Control. Fusion 53124014

[39] Fitzpatrick R 1998 Bifurcated states of a rotating tokamak plasma in the presence of a static error-field Phys. Plasmas 5 3325-41 